\title{
Overexpression of microRNA-21 inhibits the growth and metastasis of melanoma cells by targeting MKK3
}

\author{
MENG ZHOU ${ }^{1}$, XIAOQIAN YU ${ }^{2}$, ZHENHAI JING ${ }^{3}$, WEI WU ${ }^{4}$ and CHENGLONG LU ${ }^{5}$ \\ ${ }^{1}$ Department of Dermatology, Qilu Hospital of Shandong University, Qingdao, Shandong 266000; \\ Departments of ${ }^{2}$ Dermatology and ${ }^{3}$ Oncology, Qingdao Hiser Medical Group, Qingdao Hospital of \\ Traditional Chinese Medicine, Qingdao, Shandong 266032; ${ }^{4}$ College of Food Science and Technology, \\ Qingdao Agricultural University, Qingdao, Shandong 266179; ${ }^{5}$ Department of Emergency, \\ Qilu Hospital of Shandong University, Qingdao, Shandong 266000, P.R. China
}

Received November 6, 2018; Accepted May 21, 2019

DOI: $10.3892 / \mathrm{mmr} .2019 .10408$

\begin{abstract}
Melanoma is an aggressive skin carcinoma with poor prognosis, and is prevalent worldwide. It was demonstrated that microRNA (miR)-21 and mitogen-activated protein kinase kinase 3 (MKK3) both participated in the occurrence and development of various tumors; however, their detailed roles in the progression of melanoma remain unclear. Reverse transcription-quantitative PCR (RT-qPCR) and western blot analyses were conducted to examine the expression levels of miR-21 and MKK3 in clinical specimens of patients with melanoma and melanoma cell lines. A dual-luciferase reporter assay was performed to verify the target interaction between miR-21 and MKK3. The mRNA and protein expressions of MKK3 were measured using RT-qPCR and western blot analysis, respectively, following transfection with miR-21 mimics and inhibitor. Subsequently, Cell Counting Kit-8 and colony formation assays, and flow cytometry were conducted to assess the effects of miR-21 and MKK 3 on the cell growth of melanoma. Cell migration and invasion experiments were performed to evaluate the effects of miR-21 and MKK3 on the cell metastasis of melanoma. It was revealed that MKK3 was upregulated, and miR-21 was downregulated in patients with melanoma and melanoma cell lines. MKK3 was demonstrated to be a direct target of miR-21. Furthermore, it was demonstrated that upregulated miR-21 expression and downregulated MKK3 expression suppressed cell proliferation and colony formation, promoted apoptosis, delayed the cell cycle, and inhibited cell migration and invasion. The present findings suggested that miR-21 could inhibit the cell growth and metastasis of melanoma by negatively regulating MKK3.
\end{abstract}

Correspondence to: Dr Chenglong Lu, Department of Emergency, Qilu Hospital of Shandong University, 758 Hefei Road, Qingdao, Shandong 266000, P.R. China

E-mail: qlqdlcl@126.com

Key words: melanoma, microRNA-21, mitogen-activated protein kinase kinase 3 , cell growth and metastasis

\section{Introduction}

Melanoma is an aggressive form of skin cancer, which is formed by the malignant transformation of melanocytes located at the basement of the epidermis, deriving from blemishes or pigmented spots $(1,2)$. It is one of the most lethal malignancies globally, particularly in fair-skinned individuals, accounting for $80 \%$ of skin cancer-related mortality (3). Although efforts have been made to develop improved understanding of melanoma progression and more effective treatments, they have met with limited success, mainly due to its high metastatic potential and poor prognosis (4). Therefore, further investigation into the molecular mechanisms underlying the development and progression of melanoma is urgently required to design new therapeutic strategies and improve clinical outcomes. Previously, it has been found that the abnormal expression of genes, such as B-Raf and Asparaginyl-TNRA synthetase, serves an important role in the formation and metastasis of melanoma $(5,6)$. Targeted manipulation of the altered expression or regulatory mechanisms of these genes may be a potential treatment strategy (6).

MicroRNAs (miRNAs/miRs), a class of short, endogenous, noncoding RNAs ranging between 19 and 25 nucleotides in length, were found to participate in a variety of physiological and pathological activities, including cell proliferation, transformation and tumorigenesis (7). Furthermore, the function of miRNA in gene regulation is exerted primarily via binding to the untranslated region (UTR) of target genes, which leads to mRNA degradation and/or reduced translation $(8,9)$. Generally, an individual miRNA can regulate the expression of multiple target genes, and several miRNAs can synergistically act on one target gene (10). At present, several miRNAs have been demonstrated to contribute to the aggressive phenotype of melanoma cells $(11,12)$. For example, enhanced expression of miR-612 suppressed the growth, invasion and tumorigenesis of melanoma cells by targeting Espin (13); miR-637 inhibited melanoma cell proliferation by inactivating the AKT signaling pathway and induced apoptosis through regulation of Bcl-2/Bax expression by targeting phosphatidylinositol 3,4,5-triphosphate-dependent Rac exchanger 2 protein (14); and miR-143-3p inhibited the growth and invasiveness of melanoma cells by 
targeting cyclooxygenase-2, and was inversely correlated with malignant melanoma progression (15).

miR-21 is an example of an oncomiR that is upregulated in various types of cancer (16). For instance, reduced expression of miR-21 replicated the effects of celastrol on OVCAR3 cells and inhibited the PI3K/phosphorylated-Akt-NF- $\mathrm{B}$ signaling pathway in an in vitro model of ovarian carcinoma (17). In addition, miR-21 has also been reported to be involved in the formation of melanoma $(18,19)$. miR-21 was able to regulate the ERK/NF- $\mathrm{BB}$ signaling pathway to affect the proliferation, migration and apoptosis of human melanoma A375 cells by targeting protein sprout homolog 1, programmed cell death protein 4 and PTEN $(20,21)$. Mitogen-activated protein kinase kinase 3 (MKK3), a member of the dual-specificity MKK protein kinase group that belongs to the mitogen-activated protein kinase (MAPK) signaling pathway, was identified to be associated with tumor invasion and progression (22). For example, the activation of the MKK3/MKK6-p38 axis is closely related to the cell survival of MDA-MB-231 breast cancer cells (23). In the present study, the expression levels of MKK3 mRNA and protein, and miR-21 were evaluated in biopsy samples from patients with melanoma, and the functional effects of miR-21 and MKK3 on melanoma cell proliferation, apoptosis, cell cycle, migration and invasion were examined, with the interaction between miR-21 and MKK3 being further investigated.

\section{Materials and methods}

Tissue specimens. Melanoma tissues and their corresponding matched adjacent normal tissues, that were $5 \mathrm{~cm}$ away from the tumor tissue, were collected from 15 cases of patients with melanoma who did not receive cutaneous radiotherapy or chemotherapy prior to surgical resection at the Department of Oncology, Qilu Hospital of Shandong University (Qingdao, China) between January 2017 and January 2018. The cohort included 8 males and 7 females, with a median age of 51 years (range, 39-78 years). The diagnosis of melanoma was performed by two experienced pathologists on the basis of hematoxylin and eosin staining of $5-\mu \mathrm{m}$ sections conducted according to the manufacturer's protocols (cat. no. C0105; Beyotime Institute of Biotechnology) and immunohistochemistry staining performed as previously described (23), following fixation in $4 \%$ paraformaldehyde at room temperature for $2 \mathrm{~h}$. Following resection, tumor tissues were flushed with $0.9 \%$ saline, and regions with no necrosis and good vitality were selected for experiments, while the normal tissues were similarly collected. Nerves, blood vessels and connective tissues were removed. Then, all tissues were rapidly snap-frozen in liquid nitrogen and stored at $-80^{\circ} \mathrm{C}$ until use.

The present study was performed in strict accordance with the Declaration of Helsinki and approved by the Clinical Management Committee of Hunan First People's Hospital (Changsha, China). Written informed consent for research purposes was obtained from all patients. The information of the patients is presented in Table I.

Cell lines and culture. The melanoma A2058 and HS294T cell lines, skin fibroblast foreskin BJ and CCC-HSF-1 cell lines, epithelial HME1 cells from mammary glands and 293T cells
Table I. Patient information.

\begin{tabular}{lll}
\hline $\begin{array}{l}\text { Specimen } \\
\text { number }\end{array}$ & Type of melanoma & Tumor metastasis \\
\hline 1 & Primary melanoma & Yes \\
2 & Primary melanoma & Yes \\
3 & Primary melanoma & No \\
4 & Primary melanoma & No \\
5 & Primary melanoma & Yes \\
6 & Primary melanoma & No \\
7 & Primary melanoma & No \\
8 & Primary melanoma & No \\
9 & Primary melanoma & Yes \\
10 & Primary melanoma & Yes \\
11 & Primary melanoma & No \\
12 & Primary melanoma & Yes \\
13 & Primary melanoma & Yes \\
14 & Primary melanoma & No \\
15 & Primary melanoma & No
\end{tabular}

were purchased from American Type Culture Collection. 293T cells were cultured in DMEM (Gibco; Thermo Fisher Scientific, Inc.), whereas all other cell lines were cultured in RPMI-1640 medium (Thermo Fisher Scientific, Inc.), respectively, which were both supplemented with $10 \%$ FBS (Invitrogen; Thermo Fisher Scientific, Inc.), 2 mM L-glutamine, $100 \mathrm{U} / \mathrm{ml}$ penicillin and $100 \mu \mathrm{g} / \mathrm{ml}$ streptomycin (Gibco; Thermo Fisher Scientific, Inc.) at a temperature of $37^{\circ} \mathrm{C}$ in a humidified incubator with $95 \% \mathrm{O}_{2}$ and $5 \% \mathrm{CO}_{2}$. HME1 skin cells were used as controls.

RNA extraction, cDNA synthesis and reverse transcription-quantitative PCR $(R T-q P C R)$ analysis. Total RNA was obtained from tissues and all cell lines using TRIzol ${ }^{\circledR}$ reagent (Invitrogen; Thermo Fisher Scientific, Inc.) according to the manufacturer's protocol. Then, the isolated RNA was cleared of contaminating genomic DNA using DNase treatment (Thermo Fisher Scientific, Inc.) and the RT reaction was performed using a PrimeScript ${ }^{\mathrm{TM}}$ RT kit (Takara Bio, Inc.) in a PCR thermocycling instrument (Applied Biosystems; Thermo Fisher Scientific, Inc.) at $16^{\circ} \mathrm{C}$ for $30 \mathrm{~min}, 42^{\circ} \mathrm{C}$ for $30 \mathrm{~min}$ and $85^{\circ} \mathrm{C}$ for $5 \mathrm{~min}$ to obtain cDNA. Finally, the expression levels of MKK3 and miR-21 were detected using PCR amplification with a commercially available SYBR Green qPCR SuperMix kit (Invitrogen; Thermo Fisher Scientific, Inc.) on

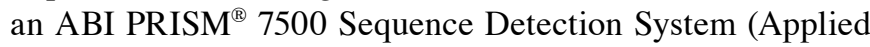
Biosystems; Thermo Fisher Scientific, Inc.) at $95^{\circ} \mathrm{C}$ for $2 \mathrm{~min}$, followed by 40 cycles of $95^{\circ} \mathrm{C}$ for $15 \mathrm{sec}$, and $60^{\circ} \mathrm{C}$ for $32 \mathrm{sec}$ (with data collection at the end of the $60^{\circ} \mathrm{C}$ step at each cycle), followed by a melting curve. GAPDH and U6 small nuclear RNA were used as the endogenous controls to quantify mRNA and miRNA expression levels, respectively. The fold changes of gene expression levels were calculated using the $2^{-\Delta \Delta C q}$ method (24). The primers used are presented in Table II.

Western blot analysis. Protein lysate preparation from human tissues and cells was performed on ice using RIPA buffer 
Table II. Sequences of the primers applied in the RT-quantitative PCR assays.

\begin{tabular}{ll} 
miR or gene & \multicolumn{1}{c}{ Primer sequences (5'-3') } \\
\hline miR-21 & F: ACACTCCAGCTGGGTAGCTTATCAGACTGA \\
& R: TGGTGTCGTGGAGTCG \\
U6 & RT: CTCAACTGGTGTCGTGGAGTCGGCAATTCAGTTGAGAAAAATATGG \\
& F: CTCGCTTCGGCAGCACA \\
& R: AACGCTTCACGAATTTGCGT \\
MKK3 & F: TGCTCATGGACCTGGACATC \\
& R: GAGGGCTTCACATCTCTGTG \\
GAPDH & F: CCTGGATACCGCAGCTAGGA \\
& R: GCGGCGCAATACGAATGCCCC
\end{tabular}

miR, microRNA; MKK3, mitogen-activated protein kinase kinase 3; RT, reverse transcription; F, forward; R, reverse.

(Beyotime Institute of Biotechnology) comprising $150 \mathrm{mM}$ $\mathrm{NaCl}, 50 \mathrm{mM}$ Tris- $\mathrm{HCl}$ ( $\mathrm{pH} 8.0), 0.5 \%$ sodium deoxycholate, $1 \% \mathrm{NP}-40$ and $0.1 \% \mathrm{SDS}$, and supplemented with $1 \mathrm{mg} / \mathrm{ml}$ aprotinin, sodium $1 \mathrm{mM}$ orthovanadate and $0.1 \mathrm{mg} / \mathrm{ml}$ phenylmethylsulfonyl fluoride. Concentrations of protein were assessed with a bicinchoninic acid protein assay kit (Thermo Fisher Scientific, Inc.). Then, the proteins $(30 \mu \mathrm{g})$ were separated on a $12 \%$ SDS-PAGE gel and transferred to a $0.22-\mu \mathrm{m}$ PVDF membrane (EMD Millipore). After blocking the membranes in 5\% skimmed milk in TBS with $0.1 \%$ Tween-20 (TBST) for $2 \mathrm{~h}$ at room temperature, the PVDF membranes were incubated in PBS containing dilutions of rabbit anti-MKK3 (1:1,500; cat. no. ab5428; Abcam) and mouse anti-GAPDH (1:2,000; cat. no. ab9484; Abcam) antibodies at $4^{\circ} \mathrm{C}$ overnight. Subsequently, the PVDF membranes were washed with TBST buffer three times, and incubated with PBS, including an anti-rabbit (cat. no. BA1056) or anti-mouse (cat. no. BA1050) secondary antibody (Wuhan Boster Biological Technology, Ltd.) conjugated to horseradish peroxidase $(1: 10,000)$ for $1 \mathrm{~h}$ at room temperature with gentle agitation. Following three washes in TBST, the membranes were incubated with ECL substrates (Beyotime Institute of Biotechnology) for $1 \mathrm{~min}$, and the Amersham Imager 600 system (GE Healthcare Life Sciences) or X-ray films (Kodak) were used to visualize the target proteins. All results were normalized to the expression of GAPDH protein using ImageJ version 1.51 software (National Institutes of Health).

Transfection and dual-luciferase reporter assay. All plasmids (pLC3) used were designed and synthesized by Sangon Biotech Co., Ltd. using sequences purchased from Guangzhou RiboBio Co., Ltd., including miR-21 mimics (cat. no. miR10000076-1-5), miR-21 inhibitor (cat. no. miR20000076-1-5a), NC inhibitor (cat. no. miR1N0000001-2-5), MKK3-overexpression plasmid and MKK3-small interfering (si) RNA (cat. no. siB08711160442-1-5). Empty pLC3 vector was used as a NC for all transfections except inhibitor transfection. A2058 cells plated in 6-well plates at a density of $2 \times 10^{5}$ cells/well were transfected with the various plasmids (1 ng) using Lipofectamine ${ }^{\circledR}$ 2000 (Invitrogen; Thermo Fisher Scientific, Inc.), according to the manufacturer's protocol. Subsequent experiments were performed $48 \mathrm{~h}$ following transfection.
The binding sequences between MKK3 and miR-21 were predicted using TargetScan version 7.2 software (25). For the dual-luciferase reporter assay, 293T cells seeded into 24-well plates were transfected with $200 \mathrm{ng}$ miR-21 mimics, miR-21 inhibitor or the corresponding control vectors along with $50 \mathrm{ng}$ psiCHECK ${ }^{\mathrm{TM}}-2$ containing wild-type (WT) or mutant 3'-UTR of MKK3 mRNA inserted downstream of Renilla luciferase (Promega Corporation). Following a 48-h incubation, the cells were harvested and assessed for Renilla and firefly luciferase signals using a dual-luciferase reporter gene detection system, according to the manufacturer's protocol (cat. no. E2920; Promega Corporation).

Cell proliferation evaluation. A2058 cells (1,000 cells/well) were treated with the indicated conditions in 96-well plates. Cell proliferation was determined using a Cell Counting Kit-8 (CCK-8) assay according to the manufacturer's protocol. At 0, 1, 2 and 3 days, $90 \mu \mathrm{l}$ fresh culture media and $10 \mu \mathrm{l} \mathrm{CCK}-8$ solution (cat. no. C0038; Beyotime Institute of Biotechnology) were added to each sample. Subsequently, transfected melanoma cells were incubated at $37^{\circ} \mathrm{C}$ for $2 \mathrm{~h}$. Finally, the absorbance was measured at $450 \mathrm{~nm}$ using a microplate reader (Bio-Rad Laboratories, Inc.).

Soft agar assay. Transfected A2058 cells (1,000 cells/well) were treated with the indicated conditions in 6-well plates. After $48 \mathrm{~h}$, the cells were trypsinized, gently mixed with $0.45 \%$ agar medium mixture and re-seeded on 6-well plates covered with a layer of $0.9 \%$ agar in DMEM. After 2 weeks, the colonies were stained with $0.2 \%$ crystal violet for $5 \mathrm{~min}$ at room temperature, and quantified by colony counting.

Apoptosis and cell cycle assessment. A2058 cells were transfected with control or miR-21 mimics, MKK3 expression vector or small interfering RNA in 6-well plates. After $48 \mathrm{~h}$ of transfection, $2 \times 10^{5}$ cells were harvested, centrifuged for $5 \mathrm{~min}$ at room temperature within $500 \mathrm{x} \mathrm{g}$, and washed three times with cold PBS. For cell apoptosis analysis, a cell apoptosis detection kit was used, according to the manufacturer's protocol (cat. no. C1062; Beyotime Institute of Biotechnology). Briefly, the harvested cells were suspended in $500 \mathrm{ml}$ binding buffer and incubated at room temperature in the dark for 
A

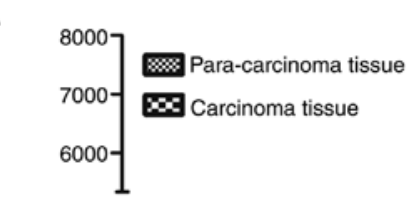

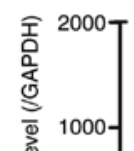

芯

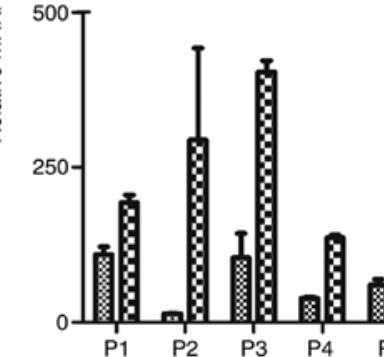

B
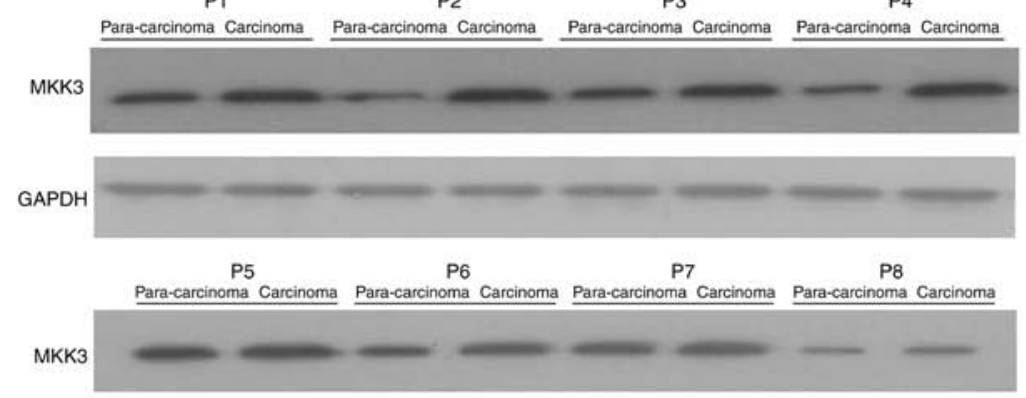

GAPDH

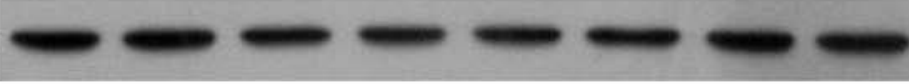

P9

P10

P11

$P 12$

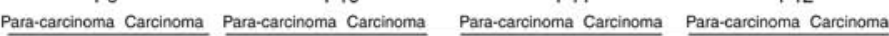

MKK3

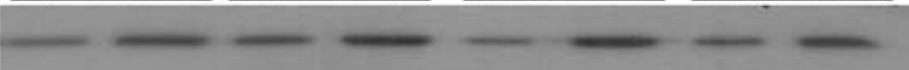

GAPDH -

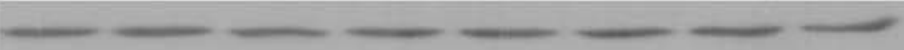

$\mathrm{P} 13$

P14

P15

Para-carcinoma Carcinoma Para-carcinoma Carcinoma Para-carcinoma Carchoma

MKK3

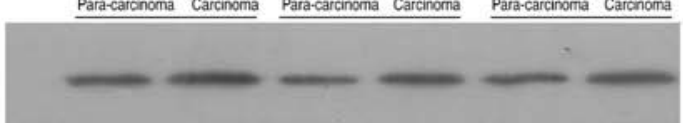

GAPDH

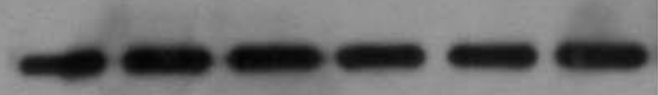

MKK3 protein expression

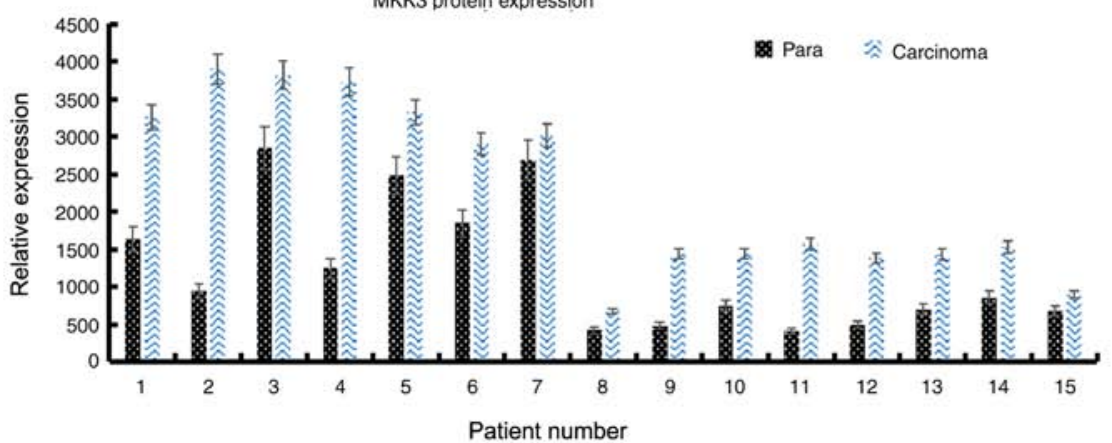

Figure 1. MKK3 and miR-21 expression levels are dysregulated in melanoma tissues. (A) RT-qPCR analysis of MKK3 mRNA levels in 15 pairs of melanoma tissues and adjacent normal tissues. (B) Western blot analysis of MKK3 protein levels in 15 pairs of melanoma tissues and adjacent normal tissues. The band intensity was determined to quantify protein expression levels. 


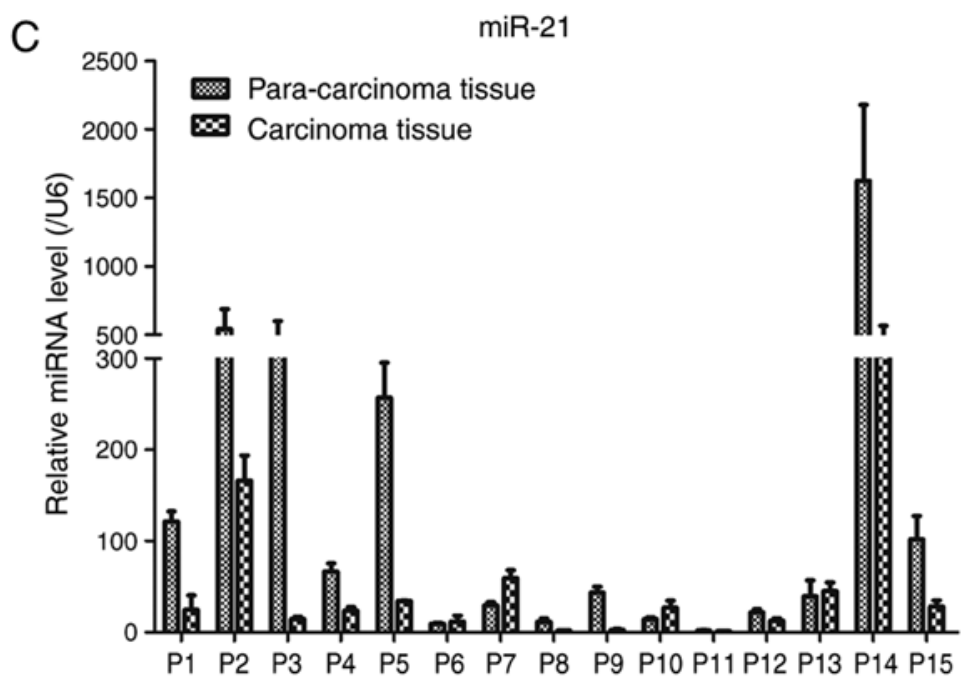

Figure 1. MKK3 and miR-21 expression levels are dysregulated in melanoma tissues. (C) RT-qPCR analysis of miR-21 levels in 15 pairs of melanoma tissues and adjacent normal tissues. The results are presented as the mean \pm standard deviation of three replicates per tissue sample. MKK3, mitogen-activated protein kinase kinase 3; miR-21, microRNA-21; RT-qPCR, reverse transcription-quantitative PCR; P, patient.
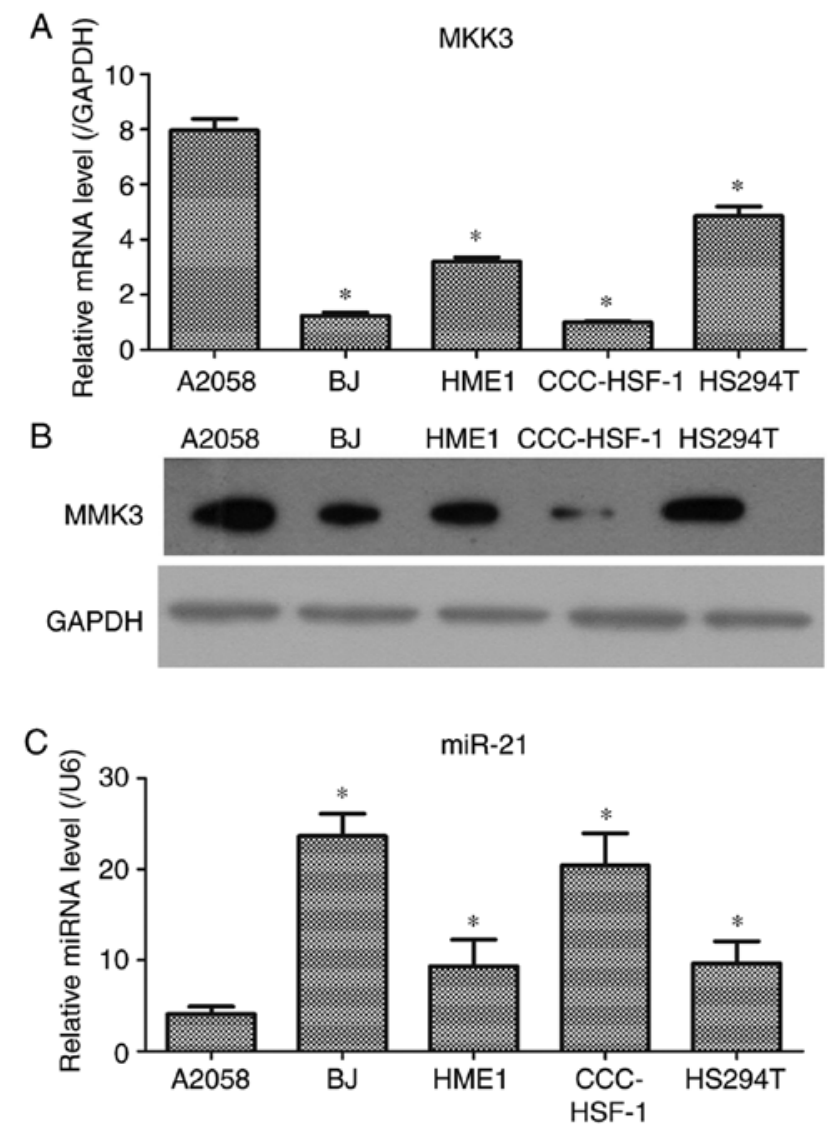

Figure 2. Quantification of MKK3 and miR-21 levels in melanoma and skin cell lines. (A) mRNA and (B) protein expression levels of MKK3, as determined via RT-qPCR and western blot analyses, respectively. (C) RT-qPCR results demonstrate the expression levels of miR-21. The results are presented as the mean \pm standard deviation of at least three independent experiments performed in triplicate. ${ }^{*} \mathrm{P}<0.05$ vs. A2058 cells. MKK3, mitogen-activated protein kinase kinase 3; miR-21, microRNA-21; RT-qPCR, reverse transcription-quantitative PCR.

15 min after being labeled with $5 \mathrm{ml}$ Annexin-V-FITC and $5 \mathrm{ml}$ propidium iodide (PI). For the cell cycle analysis, the cells were first fixed with $70 \%$ ethanol at room temperature for $1 \mathrm{~h}$, and then incubated with RNase $(50 \mu \mathrm{g} / \mathrm{ml})$ and stained with PI for $30 \mathrm{~min}$ at $4^{\circ} \mathrm{C}$ in the dark. A flow cytometer and WinMDI version 2 software (BD Biosciences) was used to evaluate apoptosis and the cell cycle. The apoptosis rate was calculated as the percentage of cells in early + late apoptosis.

Cell migration and cell invasion assays. The migration and invasion of A2058 cells were quantitatively measured using a Transwell chamber assay (Costar; Corning, Inc.). The cells $\left(5 \times 10^{4}\right.$ cells) treated with indicated conditions were seeded on the upper insert coated without or with $2 \%$ Matrigel (Corning, Inc.) in 24-well plates for migration or invasion assays, respectively. Subsequently, the lower chamber was filled with $600 \mu 1$ DMEM supplemented with $10 \%$ FBS as a chemoattractant, followed by an incubation at $37^{\circ} \mathrm{C}$ with $5 \% \mathrm{CO}_{2}$ for $24 \mathrm{~h}$. Next, the cells were permeated with $100 \%$ methanol for $15 \mathrm{~min}$ at room temperature, stained with $0.1 \%$ crystal violet for $20 \mathrm{~min}$ at room temperature, washed with PBS three times and dried at $80^{\circ} \mathrm{C}$ for $30 \mathrm{~min}$. Non-migrated or non-invaded cells on the inside of the wells were removed with a cotton swab, and the the cells were counted in images captured in five random fields under a light microscope (magnification, x200; Olympus Corporation).

Statistical analysis. Statistical calculations were performed using IBM SPSS software, version 23.0 (IBM Corp.). Linear regression analysis of MKK3 and miR-21 expression in melanoma tissue was conducted using Microsoft Excel 2010 (Microsoft Corporation). All cell experiments were independently repeated at least three times and all data are presented as the mean \pm standard deviation. Protein and mRNA expression values in individual patient tissues are presented as the mean \pm standard deviation of three replicates. Any differential expression between two groups was determined using an independent Student's t-test. Statistical comparisons among multiple groups were performed using Tukey's one-way analysis of variance. $\mathrm{P}<0.05$ was considered to indicate a statistically significant difference. 


\section{A Wild type MKKз 3'-UTR 5'-GUGCUACCCACACCAUAAGCUA-3' \\ miR-21 3'-AGUUGUAGUCAGACUAUUCGAU-5' \\ Mutant MKK3 3'-UTR $\quad$ 5'-GUGCUACCCACACCAGCTCCAT-3'}
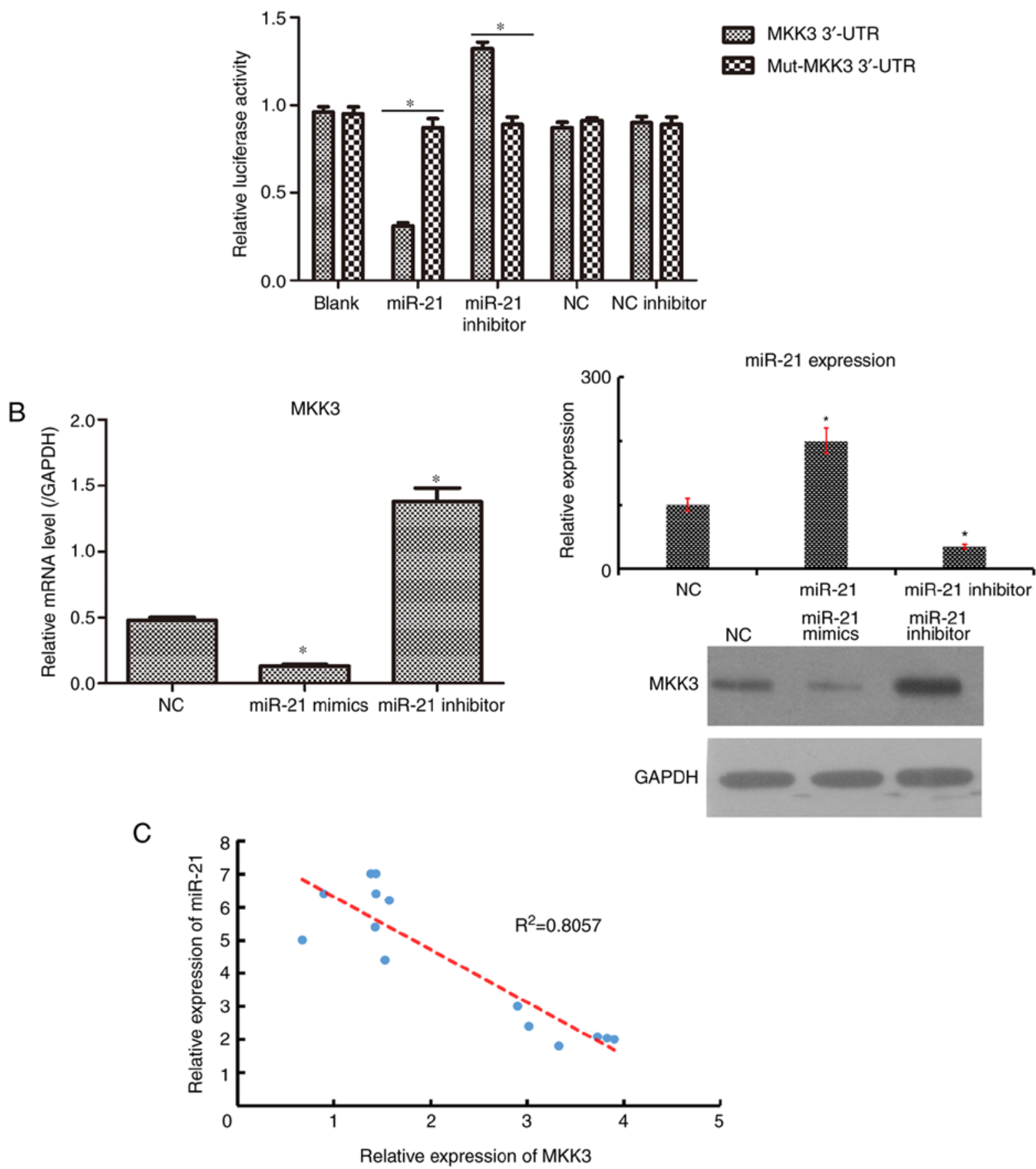

Figure 3. miR-21 directly targets MKK3. (A) Alignment of the interaction sites in the human 3'-UTR of MKK3 gene and the mutated 3'-UTR of MKK3 is highlighted in red. The target interaction between miR-21 and MKK3 was tested using a dual-luciferase assay in $293 \mathrm{~T}$ cells. "P<0.05. (B) Reverse transcription-quantitative PCR and western blot analyses were used to examine the expression levels of MKK3 and miR-21 in A2058 cells, following transfection with miR-21 mimic and inhibitor. " $\mathrm{P}<0.05$ vs. NC. (C) Linear regression of MKK3 expression and miR-21 expression in the 15 pairs of tumor tissues. The results are presented as the mean \pm standard deviation of at least three independent experiments performed in triplicate. MKK3, mitogen-activated protein kinase kinase 3; miR-21, microRNA-21; UTR, untranslated region; NC, negative control; mut, mutant.

\section{Results}

Upregulation of $M K K 3$ and downregulation of miR-21 in melanoma. Expression of MKK3 and miR-21 in melanoma was evaluated via RT-qPCR in 15 pairs of human primary melanoma specimens and corresponding adjacent skin. The protein expression levels of MKK3 in the tissues were also assessed using western blot analysis. The results demonstrated that MKK3 mRNA was upregulated and MKK3 protein was overexpressed in melanoma biopsy samples 
A
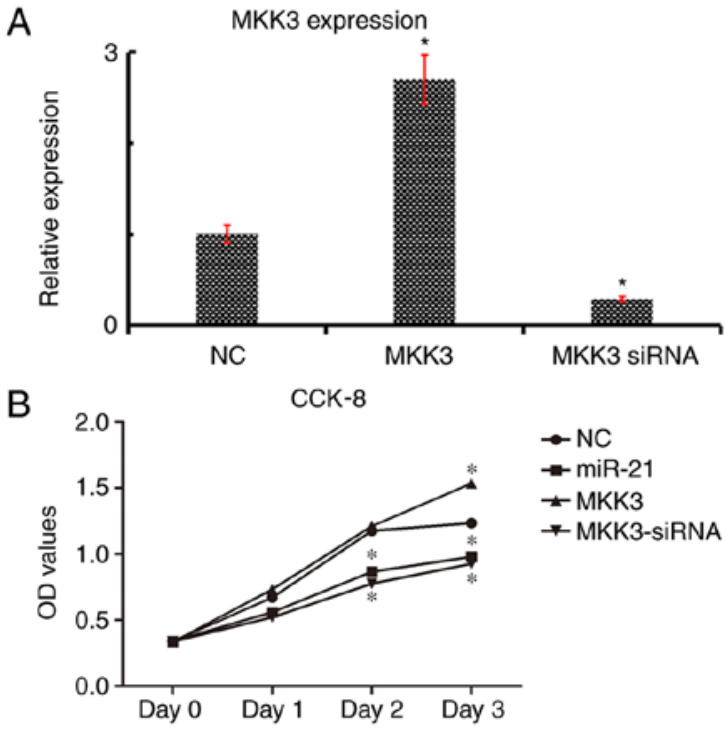

C
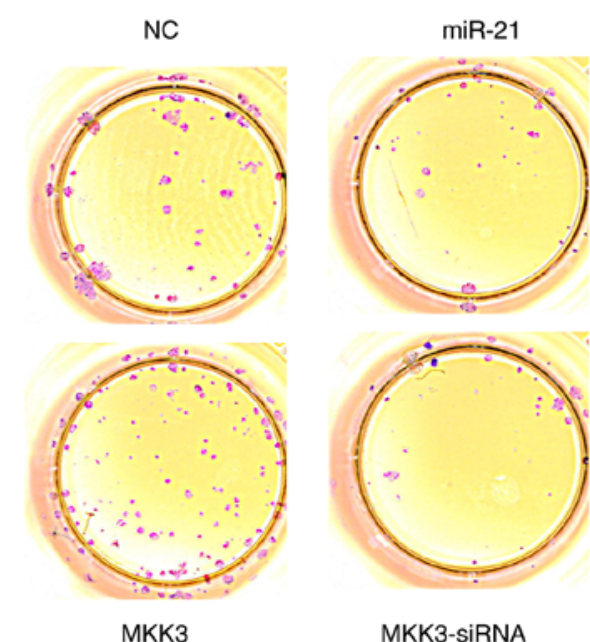

MKK3-siRNA

Figure 4. Restoration of miR-21 reduces, and overexpression of MKK3 induces proliferation and tumorigenesis. (A) Expression of MKK3 in transfected A2058 cells. ${ }^{*} \mathrm{P}<0.05$ vs NC. (B) A2058 cell proliferation was analyzed using a CCK- 8 assay. ${ }^{*} \mathrm{P}<0.05$ vs. NC. (C) Representative images of the colony formation assay. MKK3, mitogen-activated protein kinase kinase 3; miR-21, microRNA-21; NC, negative control; CCK-8, Cell Counting Kit-8; OD, optical density; siRNA, small interfering RNA.

compared with their corresponding adjacent normal tissue samples (Fig. 1A and B). Conversely, the miR-21 expression profile was reversed compared with the MKK3 expression in these tissues, which indicated that miR-21 expression was lower in primary melanoma tissues compared with the matched adjacent healthy tissues. The mRNA expression of miR-21 was also determined (Fig. 1C). Therefore, these findings indicated that MKK3 was upregulated, and miR-21 was downregulated in melanoma.

MKK3 is significantly increased and miR-21 is decreased in melanoma cells. The expression levels of MKK3 and miR-21 in melanoma cells were further examined. The results demonstrated that the mRNA and protein levels of MKK3 in A2058 cells were significantly increased compared with HS294T melanoma cells and the control skin cells (Fig. 2A and B). It was also observed that miR-21 expression in A2058 cells was

significantly decreased compared with that in HS294T melanoma cells and the control skin cells (Fig. 2C). Therefore, these data indicated that MKK3 may be enhanced and miR-21 may be downregulated in A2058 cells, which was similar to the results from the clinical specimens; A2058 cells were selected for the following experiments, as the cell line exhibited the highest MMK3 and lowest miR-21 expression levels.

$M K K 3$ is a novel target gene of $m i R-21$. Based on the inverse expression profiles of MKK3 and miR-21, the underlying interaction between MKK3 and miR-21 required investigation. First, TargetScan predicted that MKK3 was a potential target gene of miR-21 (25). Cells were transfected with plasmids containing the WT and mutant 3'-UTR of MKK3 with miR-21. A luciferase reporter assay was conducted to confirm that miR-21 could directly target the 3'-UTR of MKK3. As presented in Fig. 3A, the forced expression of miR-21 decreased the luciferase activity in 293T cells with the WT plasmid $(\mathrm{P}<0.05)$, although no significant change was observed in cells transfected with the mutant plasmid. To further detect whether miR-21 could affect MKK3 expression in melanoma cells, RT-qPCR and western blot analyses were conducted to quantify MKK3 mRNA and protein expression following transfection with miR-21 mimics and miR-21 inhibitor in A2058 cells. It was observed that the upregulation of miR-21 suppressed MKK3 expression at the mRNA and protein levels, whereas the downregulation of miR-21 promoted MKK3 expression at the mRNA and protein levels, compared with NC plasmid (Fig. 3B). In addition, the expression of MKK3 in 15 melanoma tissues was negatively correlated with miR-21 expression (Fig. 3C). Thus, these results collectively suggested that MKK3 may be a direct target of miR-21.

MKK3 mitigates miR-21 stimulation of proliferation in melanoma cells. To explore the functions of MKK3 and miR-21 in A2058 cells, miR-21 was overexpressed by transfecting with miR-21 mimics, and MKK3 was overexpressed by transfecting with an MKK3 overexpression vector. MKK3 expression was significantly increased in A2058 cells following transfection with the MKK3 vector; conversely, expression was significantly downregulated following transfection with MKK3 siRNA (Fig. 4A) Initially, the roles of miR-21 and MKK3 in cell growth were investigated; compared with the empty vector NC group, miR-21 overexpression and anti-MKK3 expression markedly inhibited the growth of melanoma cells, as determined by CCK-8 and soft agar assays (Fig. 4B and C). Additionally, it was demonstrated that MKK3 overexpression accelerated the growth of melanoma cells (Fig. 4B and C). Therefore, these data suggested that miR-21 might restrain cell growth and MKK3 might stimulate the cell growth of melanoma cells.

miR-21 activates melanoma cell apoptosis and causes cell cycle arrest, but MKK3 plays an inverse role. The effects of miR-21 and MKK3 on cell apoptosis and cell cycle distribution were assessed. It was revealed that enhanced expression of miR-21 and reduced expression of MKK3 significantly aggravated the percentage of cells undergoing apoptosis and arrested the cell cycle at the $\mathrm{G}_{1}$ phase compared with the $\mathrm{NC}$ 
A

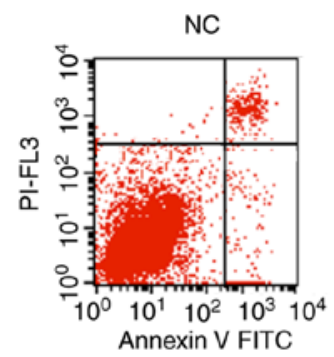

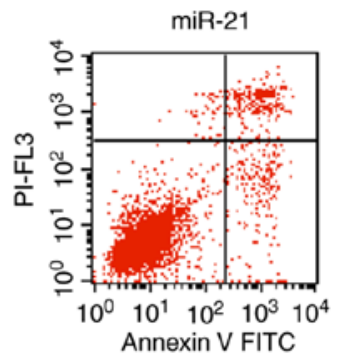
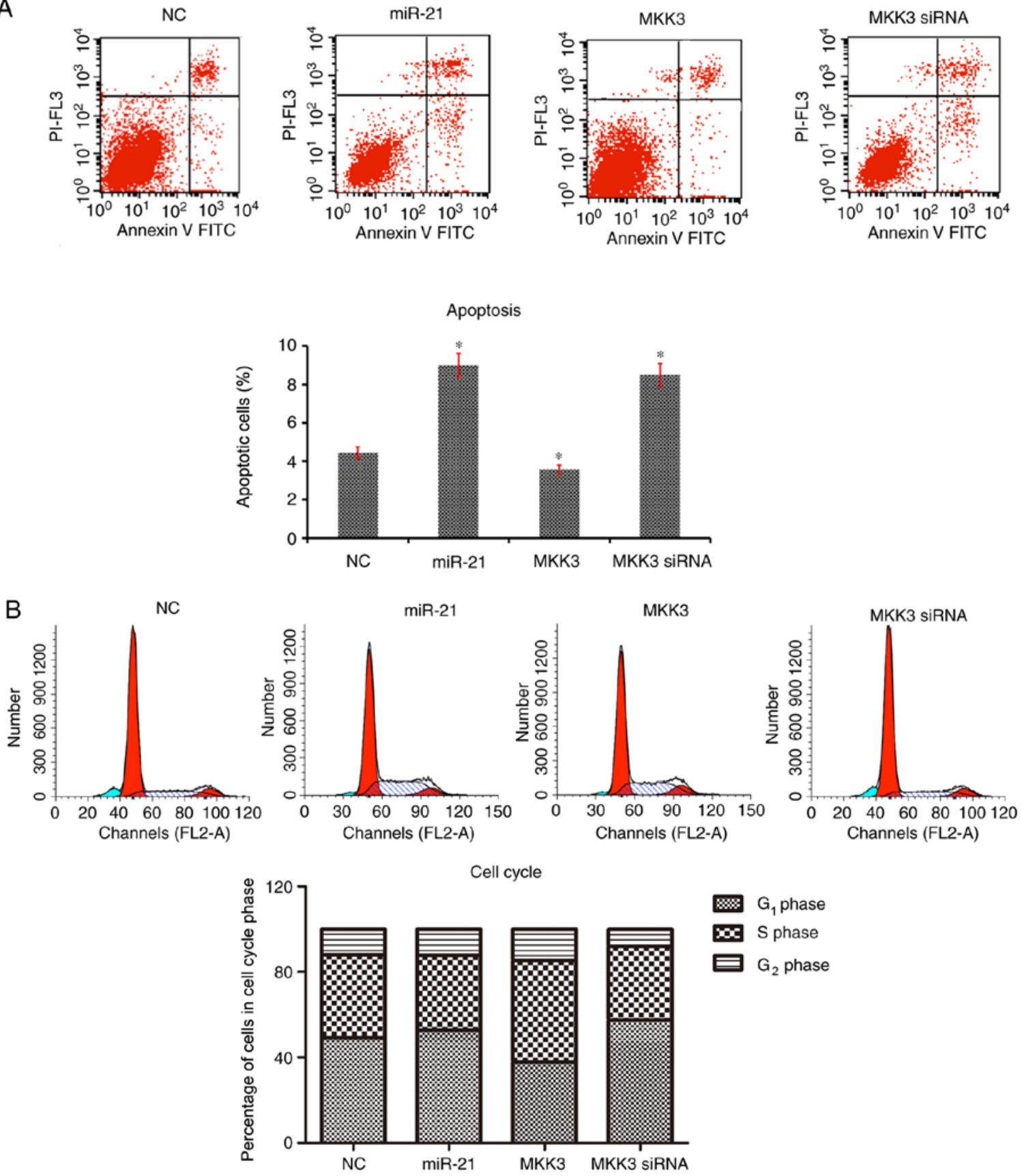

Figure 5. miR-21 upregulation and MKK3 downregulation enhances A2058 cell apoptosis and delays the cell cycle. (A) Percentage apoptosis was analyzed using flow cytometry. (B) Cell cycle distribution was analyzed using flow cytometry. The results are presented as the mean \pm standard deviation of at least three independent experiments performed in triplicate. ${ }^{~} \mathrm{P}<0.05$ vs. NC. MKK3, mitogen-activated protein kinase kinase 3; miR-21, microRNA-21; PI, propidium iodide; siRNA, small interfering RNA; NC, negative control.

(Fig. 5A and B). Nevertheless, overexpression of MKK3 did not elevate the cell apoptosis percentage or delay the cell cycle in $\mathrm{G}_{1}$ phase (Fig. 5A and B). Taken together, these results suggested that miR-21 may stimulate melanoma cell apoptosis and contributed to cell cycle arrest in $\mathrm{G}_{1}$ phase, and MKK3 exerted an opposite effect on the apoptosis and cell cycle of melanoma cells.

miR-21 expression suppresses, and MKK3 upregulation potentiates the migration and invasion of melanoma cells. As it was observed that the expression of miR-21 and MKK3 were closely associated with melanoma features, it was hypothesized that miR-21 and MKK3 may serve an important role in its capacity to metastasize. To test this hypothesis, a Transwell chamber was used to quantify the changes in cell migration and invasion. It was demonstrated that both miR-21 mimics- and MKK3-siRNA-transfected A2058 cells exhibited significantly reduced migratory and invasive abilities compared with NC cells (both $\mathrm{P}<0.01$; Fig. 6A and B). Nevertheless, MKK3-overexpressing melanoma cells exhibited greater migratory and invasive abilities than the $\mathrm{NC}$ cells (both $\mathrm{P}<0.01$; Fig. 6A and B). Taken together, these in vitro findings concluded that miR-21 might inhibit tumor cell migration and invasion, whereas MKK3 might induce tumor cell migration and invasion. 
A

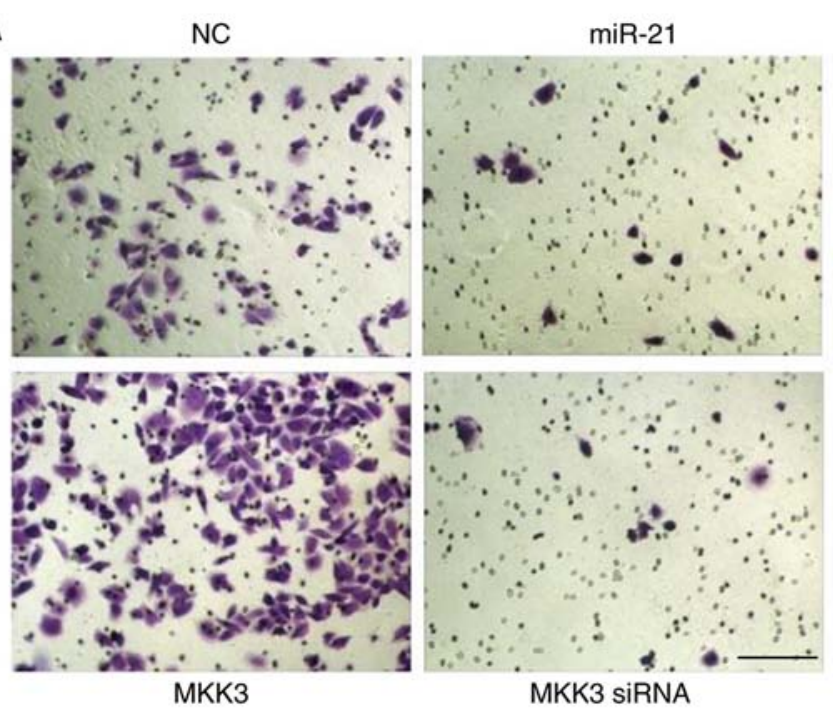

B

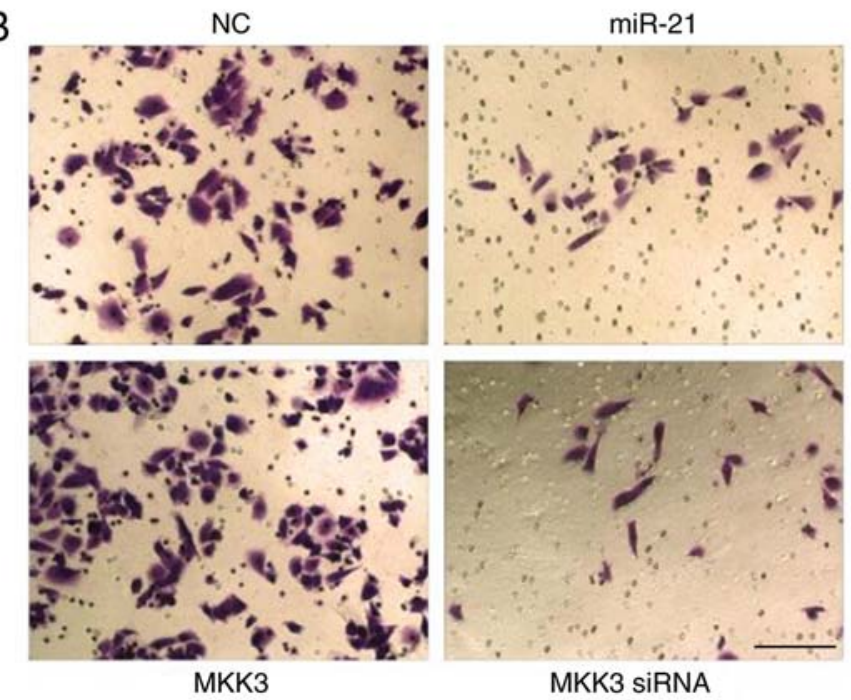

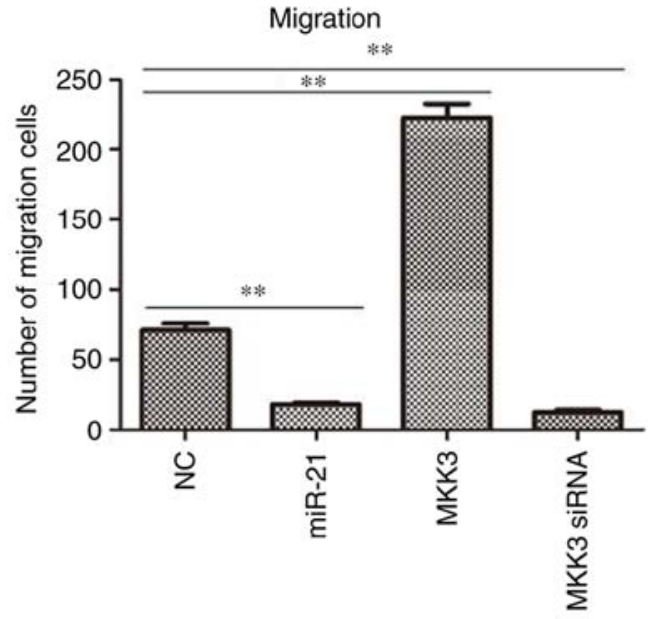

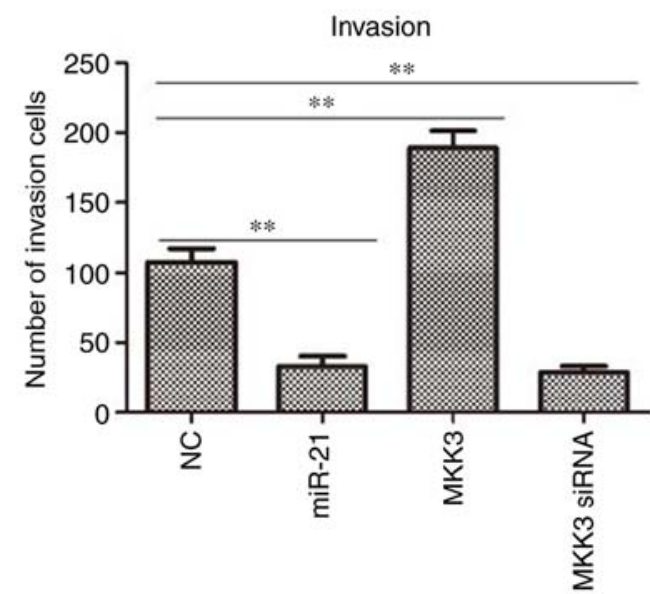

Figure 6. miR-21 inhibits, and MKK3 promotes melanoma cell migration and invasion. (A) Migratory ability of A2058 cells was analyzed using a Transwell assay. (B) Invasive ability of cells was compared using a Matrigel assay. Scale bar, $20 \mu \mathrm{m}$ (magnification, $\mathrm{x} 200$ ). ${ }^{* * *} \mathrm{P}<0.01$. The results are presented as the mean \pm standard deviation of at least three independent experiments performed in triplicate. MKK3, mitogen-activated protein kinase kinase 3; miR-21, microRNA-21; NC, negative control; siRNA, small interfering RNA.

\section{Discussion}

Melanoma is the most lethal tumor derived from melanocytes found predominantly in the skin (2). A previous epidemiological study has reported that the global incidence of melanoma has increased over the past two decades (26). The tumorigenesis and progression of melanoma is a complex and multistep process that results from interactions between chromosomal abnormalities, translocations, gene mutations, epigenetic changes and environmental factors $(6,27)$. Patients who acquire melanoma at an early stage could be treated with surgical management; however, patients at advanced stages respond poorly to current treatments $(28,29)$. Although the understanding of the molecular mechanisms involved in the pathogenesis of melanoma and the myriad of treatment studies have rapidly advanced over the past two decades, effective early diagnostic technologies and systemic therapeutic strategies of this disease remain unsatisfactory, presenting a five-year survival rate $<15 \%$ (30). Therefore, effective management of melanoma depends heavily on early diagnosis and further research mainly focuses on the identification of new high sensitivity and specificity biomarkers that are involved in the initiation, promotion and progression of melanoma (12).

MKK3, a member of the dual specificity protein kinase group that belongs to the MAPK kinase family, has a notable role in the invasion and progression of gliomas and breast tumors; it is a promising target for anticancer therapies (31). In the present study, it was demonstrated that the mRNA and protein expression levels of MKK3 were increased in melanoma patients and cell lines. It was additionally observed that the miR-21 expression was decreased in these samples. A previous study suggested that miR-21, as an oncogenic miRNA, could regulate cancer cell proliferation, migration and apoptosis by suppressing the expression of tumor suppressors (16). For example, miR-21 can modulate malignant phenotypes, 
including proliferation, anti-apoptosis, cell cycle progression and invasion of colorectal cancer cells by downregulating PTEN protein expression (32). One possible explanation for this discrepancy is that miR-21 serves its biological roles in a tissue/developmental stage-specific context. Thus, these results suggested that the abnormal expressions of MKK3 and miR-21 might play an important role in the progression of melanoma.

Based on the opposing expression profiles of MKK3 and miR-21, it was further hypothesized that there was a target relationship between MKK3 and miR-21. Subsequently, this hypothesis was verified by a dual-luciferase reporter assay. In order to investigate the influences of MKK3 and miR-21 on the phenotype of melanoma cells, cell proliferation, apoptosis, the cell cycle, migration and invasion were examined by transfecting melanoma cells with miR-21 mimics, MKK3 mimics and MKK3-siRNA. Cell growth is strictly regulated by cell proliferation and apoptosis, as well as the cell cycle (33). Dysregulation of cell proliferation, apoptosis and the cell cycle, induced by miRNA changes, is often implicated in the occurrence and development of cancer (34). In the present study, upregulation of miR-21 inhibited cell growth, and knockdown of MKK3 expression had similar effects to the miR-21 mimics group on cell growth in melanoma cells. Additionally, accumulating evidence has suggested that metastasis is the final stage of the deterioration process in tumor development for decades $(35,36)$. Furthermore, metastasis causes $\sim 90 \%$ of all cancer fatalities and mainly contains two vital steps, namely migration and invasion (37). In the present study, the results demonstrated that overexpression of miR-21 and reduced expression of MKK3 markedly decreased the number of migratory and invasive cells, but enhanced expression of MKK3 induced opposing effects on the migration and invasion of melanoma cells.

In conclusion, in the present study, it was demonstrated for the first time, to the best of our knowledge, that MKK3 and miR-21 were abundantly expressed in patients with melanoma and melanoma cell lines. Additionally, the abnormal expression of these two demonstrated an inverse association, and it was further confirmed using a dual-luciferase reporter assay that miR-21 could directly target the 3'-UTR of MKK3. Furthermore, it was also revealed that miR-21 could regulate cell biological behaviors, including cell proliferation, colony formation, apoptosis, cell cycle, cell migration and invasion, by negatively interacting with MKK3. Collectively, these findings may provide novel insight for melanoma diagnosis and prognosis, as well as therapeutic strategy.

\section{Acknowledgements}

Not applicable.

\section{Funding}

No funding was received.

\section{Availability of data and materials}

The datasets used and/or analyzed during the current study are available from the corresponding author upon reasonable request.

\section{Authors' contributions}

MZ and CL conceived and designed the study, collected the clinical data and drafted the manuscript. ZJ and XY performed the cellular experiments. WW interpreted the data and revised the manuscript.

\section{Ethics approval and consent to participate}

The present study was performed in strict accordance with the Helsinki Declaration and was approved by the Clinical Management Committee of Hunan First People's Hospital (Changsha, China). Written informed consent for research purposes was obtained from all the patients.

\section{Patient consent for publication}

Not applicable.

\section{Competing interests}

The authors declare that they have no competing interests.

\section{References}

1. Owens B: Melanoma. Nature 515 (Suppl): S109, 2014

2. Mohammadpour A, Derakhshan M, Darabi H, Hedayat P and Momeni M: Melanoma: Where we are and where we go. J Cell Physiol 234: 3307-3320, 2019.

3. Little EG and Eide MJ: Update on the current state of melanoma incidence. Dermatol Clin 30: 355-361, 2012.

4. Ko JS: The immunology of melanoma. Clin Lab Med 37: 449-471, 2017.

5. Hayward NK, Wilmott JS, Waddell N, Johansson PA, Field MA, Nones K, Patch AM, Kakavand H, Alexandrov LB, Burke H, et al: Whole-genome landscapes of major melanoma subtypes. Nature 545: 175-180, 2017.

6. Wong CW, Fan YS, Chan TL, Chan AS, Ho LC, Ma TK, Yuen ST and Leung SY; Cancer Genome Project: BRAF and NRAS mutations are uncommon in melanomas arising in diverse internal organs. J Clin Pathol 58: 640-644, 2005.

7. Mohr AM and Mott JL: Overview of microRNA biology. Semin Liver Dis 35: 3-11, 2015.

8. Ha M and Kim VN: Regulation of microRNA biogenesis. Nat Rev Mol Cell Biol 15: 509-524, 2014.

9. Fuchs Wightman F, Giono LE, Fededa JP and de la Mata M: Target RNAs strike back on MicroRNAs. Front Genet 9: 435, 2018.

10. Treiber T, Treiber N and Meister G: Regulation of microRNA biogenesis and its crosstalk with other cellular pathways. Nat Rev Mol Cell Biol 20: 5-20, 2019.

11. Mumford SL, Towler BP, Pashler AL, Gilleard O, Martin Y and Newbury SF: Circulating MicroRNA biomarkers in melanoma: Tools and challenges in personalised medicine. Biomolecules 8: E21, 2018.

12. Mirzaei H, Gholamin S, Shahidsales S, Sahebkar A, Jaafari MR, Mirzaei HR, Hassanian SM and Avan A: MicroRNAs as potential diagnostic and prognostic biomarkers in melanoma. Eur J Cancer 53: 25-32, 2016.

13. Zhu Y, Zhang HL, Wang QY, Chen MJ and Liu LB: Overexpression of microRNA-612 restrains the growth, invasion, and tumorigenesis of melanoma cells by targeting espin. Mol Cells 41: 119-126, 2018.

14. Zhang J, Liu WL, Zhang L, Ge R, He F, Gao TY, Tian Q, Mu X, Chen LH, Chen W and Li X: MiR-637 suppresses melanoma progression through directly targeting P-REX2a and inhibiting PTEN/AKT signaling pathway. Cell Mol Biol (Noisy-le-grand) 64: 50-57, 2018.

15. Panza E, Ercolano G, De Cicco P, Armogida C, Scognamiglio G, Botti G, Cirino G and Ianaro A: MicroRNA-143-3p inhibits growth and invasiveness of melanoma cells by targeting cyclooxygenase- 2 and inversely correlates with malignant melanoma progression. Biochem Pharmacol 156: 52-59, 2018. 
16. Pfeffer SR, Yang $\mathrm{CH}$ and Pfeffer LM: The role of miR-21 in cancer. Drug Dev Res 76: 270-277, 2015.

17. Zhang H, Li J, Li G and Wang S: Effects of celastrol on enhancing apoptosis of ovarian cancer cells via the downregulation of microRNA21 and the suppression of the PI3K/Akt-NF- $\kappa$ B signaling pathway in an in vitro model of ovarian carcinoma. Mol Med Rep 14: 5363-5368, 2016.

18. Mu Z and Sun Q: Cantharidin inhibits melanoma cell proliferation via the miR21mediated PTEN pathway. Mol Med Rep 18: 4603-4610, 2018.

19. Wandler A, Riber-Hansen R, Hager H, Hamilton-Dutoit SJ, Schmidt H, Nielsen BS, Stougaard $M$ and Steiniche T: Quantification of microRNA-21 and microRNA-125b in melanoma tissue. Melanoma Res 27: 417-428, 2017.

20. Mao XH, Chen M, Wang Y, Cui PG, Liu SB and Xu ZY: MicroRNA-21 regulates the ERK/NF- $\kappa B$ signaling pathway to affect the proliferation, migration, and apoptosis of human melanoma A375 cells by targeting SPRY1, PDCD4, and PTEN. Mol Carcinog 56: 886-894, 2017.

21. Zhang HL, Si LB, Zeng A, Long F, Qi Z, Zhao R and Bai M: MicroRNA-21 antisense oligonucleotide improves the sensitivity of human melanoma cells to cisplatin: An in vitro study. J Cell Biochem 119: 3129-3141, 2018

22. Bossi G: MKK3 as oncotarget. Aging (Albany NY) 8: 1-2, 2016

23. Huth HW, Albarnaz JD, Torres AA, Bonjardim CA and Ropert C: MEK2 controls the activation of MKK3/MKK6-p38 axis involved in the MDA-MB-231 breast cancer cell survival: Correlation with cyclin D1 expression. Cell Signal 28: 1283-1291, 2016.

24. Livak KJ and Schmittgen TD: Analysis of relative gene expression data using real-time quantitative PCR and the 2(-Delta Delta C(T)) method. Methods 25: 402-408, 2001.

25. Grimson A, Farh KK, Johnston WK, Garrett-Engele P, Lim LP and Bartel DP: MicroRNA targeting specificity in mammals: Determinants beyond seed pairing. Mol Cell 27: 91-105, 2007.

26. Fattouh K, Ducroux E, Decullier E, Kanitakis J, Morelon E, Boissonnat P, Sebbag L, Jullien D and Euvrard S: Increasing incidence of melanoma after solid organ transplantation: A retrospective epidemiological study. Transpl Int 30: 1172-1180, 2017.

27. Lee JA: Current evidence about the causes of malignant melanoma. Prog Clin Cancer 6: 151-161, 1975.
28. Wikstrom JD, Lundeberg L, Frohm-Nilsson M and Girnita A: Differences in cutaneous melanoma treatment and patient satisfaction. PLoS One 13: e0205517, 2018.

29. Marcell Szasz A, Malm J, Rezeli M, Sugihara Y, Betancourt LH, Rivas D, Gyorffy B and Marko-Varga G: Challenging the heterogeneity of disease presentation in malignant melanoma-impact on patient treatment. Cell Biol Toxicol 39: 1-14, 2019.

30. Rockberg J, Amelio JM, Taylor A, Jörgensen L, Ragnhammar P and Hansson J: Epidemiology of cutaneous melanoma in Sweden-Stage-specific survival and rate of recurrence. Int J Cancer 139: 2722-2729, 2016.

31. Baldari S, Ubertini V, Garufi A, D'Orazi G and Bossi G: Targeting MKK3 as a novel anticancer strategy: Molecular mechanisms and therapeutical implications. Cell Death Dis 6: e1621, 2015.

32. Wu Y, Song Y, Xiong Y, Wang X, Xu K, Han B, Bai Y, Li L, Zhang Y and Zhou L: MicroRNA-21 (Mir-21) promotes cell growth and invasion by repressing tumor suppressor PTEN in colorectal cancer. Cell Physiol Biochem 43: 945-958, 2017.

33. Lynch MP, Nawaz S and Gerschenson LE: Evidence for soluble factors regulating cell death and cell proliferation in primary cultures of rabbit endometrial cells grown on collagen. Proc Natl Acad Sci USA 83: 4784-4788, 1986.

34. Broussard L, Howland A, Ryu S, Song K, Norris D, Armstrong CA and Song PI: Melanoma cell death mechanisms. Chonnam Med J 54: 135-142, 2018.

35. Miller FR: Immune mechanisms in the sequential steps of metastasis. Crit Rev Oncog 4: 293-311, 1993.

36. Scully OJ, Bay BH, Yip G and Yu Y: Breast cancer metastasis. Cancer Genomics Proteomics 9: 311-320, 2012

37. Wu J, Zhang Y, Cheng R, Gong W, Ding T, Zhai Q, Wang Y, Meng B and Sun B: Expression of epithelial-mesenchymal transition regulatorsTWIST, SLUG and SNAIL in follicular thyroid tumours may relate to widely invasive, poorly differentiated and distant metastasis. Histopathology 74: 780-791, 2019.

This work is licensed under a Creative Commons Attribution-NonCommercial-NoDerivatives 4.0 International (CC BY-NC-ND 4.0) License. 